\title{
Circuit elements with memory: memristors, memcapacitors and meminductors
}

\author{
Massimiliano Di Ventra, Yuriy V. Pershin, and Leon O. Chua, Fellow, IEEE
}

\begin{abstract}
We extend the notion of memristive systems to capacitive and inductive elements, namely capacitors and inductors whose properties depend on the state and history of the system. All these elements show pinched hysteretic loops in the two constitutive variables that define them: current-voltage for the memristor, charge-voltage for the memcapacitor, and current-flux for the meminductor. We argue that these devices are common at the nanoscale where the dynamical properties of electrons and ions are likely to depend on the history of the system, at least within certain time scales. These elements and their combination in circuits open up new functionalities in electronics and they are likely to find applications in neuromorphic devices to simulate learning, adaptive and spontaneous behavior.
\end{abstract}

Index Terms-Memory, Resistance, Capacitance, Inductance, Dynamic response, Hysteresis.

\section{INTRODUCTION}

$\mathbf{C}$ IRCUITS elements that store information without the need of a power source would represent a paradigm change in electronics, allowing for low-power computation and storage. In addition, if that information spans a continuous range of values analog computation may replace the present digital one. Such a concept is also likely to be at the origin of the workings of the human brain and possibly of many other mechanisms in living organisms so that such circuit elements may help us understand adaptive and spontaneous behavior, or even learning.

One such circuit element is the memory-resistor (memristor for short) which was postulated by Chua in 1971 by analyzing mathematical relations between pairs of fundamental circuit variables [1]. The memristor is characterized by a relation between the charge and the flux, defined mathematically as the time integral of the voltage, which need not have a magnetic flux interpretation. This relation can be generalized to include any class of two-terminal devices (which are called memristive systems) whose resistance depends on the internal state of the system [2].

Many systems belong to this class, including the thermistor [3] (whose internal state depends on the temperature), molecules whose resistance changes according to their atomic configuration [4], or spintronic devices whose resistance varies

M. Di Ventra is with the Department of Physics, University of California, San Diego, La Jolla, California 92093-0319

e-mail: diventra@physics.ucsd.edu.

Yu. V. Pershin is with the Department of Physics and Astronomy and USC Nanocenter, University of South Carolina, Columbia, SC, 29208 e-mail: pershin@physics.sc.edu.

L. O. Chua is with the Department of Electrical Engineering and Computer Science, University of California, Berkeley, California, 94720 e-mail: chua@eecs.berkeley.edu.

Manuscript received January XX, 2009; revised January YY, 2009.

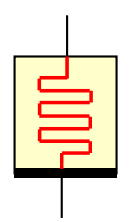

Memristor

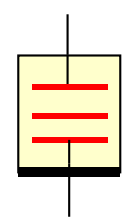

Memcapacitor

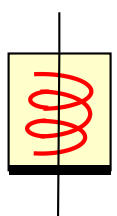

Meminductor
Fig. 1. (Color online) Symbols of the three different devices defined in this work: memristor, memcapacitor, and meminductor. In general, these devices are asymmetric and we use the following convention when possible: when a positive voltage is applied to the second (upper) terminal with respect to the terminal denoted by the black thick line, the memory device goes into a state of high resistance, capacitance or inductance, respectively.

according to their spin polarization [5], [6]. Recently, memristive behavior and memory storage have been reported in solid-state $\mathrm{TiO}_{2}$ thin films [7], [8], where the change in resistance is realized by the ionic motion of oxygen vacancies activated by current flow. Additionally, memristive behavior has been demonstrated in $\mathrm{VO}_{2}$ thin films, where the memory mechanism is related to the insulator-to-metal transition in these structures [9]. Finally, memristive behavior has been identified by two of the present authors (MDV and YVP) as a possible mechanism in the adaptive behavior of unicellular organisms such as amoebas [10].

All these examples show the ubiquitous nature of memristive systems. In fact, it should not come as a surprise that many of the above examples refer to nanoscale systems, whose resistance is likely to depend on their state and dynamical history, at least within (possibly very short) times scales dictated by the fundamental state variables that control their operation [5].

In this paper we show that the above concept of memory device is not necessarily limited to resistances but can in fact be generalized to capacitative and inductive systems. Quite generally, if $x$ denotes a set of $n$ state variables describing the internal state of the system, $u(t)$ and $y(t)$ are any two complementary constitutive variables [11] (i.e., current, charge, voltage, or flux) denoting input and output of the system, and $g$ is a generalized response, we can define a general class of $n$ th-order $u$-controlled memory devices as those described by the following relations

$$
\begin{aligned}
y(t) & =g(x, u, t) u(t) \\
\dot{x} & =f(x, u, t)
\end{aligned}
$$


where $f$ is a continuous $n$-dimensional vector function, and we assume on physical grounds that, given an initial state $u\left(t=t_{0}\right)$ at time $t_{0}$, Eq. (2) admits a unique solution [12].

Memcapacitive and meminductive systems are special cases of Eqs. (1) and (2), where the two constitutive variables that define them are charge and voltage for the memcapacitance, and current and flux for the meminductance (see Fig. 1 for the symbols we use for these devices). In this paper, we will discuss several properties of these systems which make them unique compared to their "standard" definitions and will provide few examples. Combined with the general class of memristive systems and/or standard circuit elements these devices may lead to new functionalities in electronics.

\section{MEMRISTIVE SYSTEMS}

For completeness, let us introduce first the notion of memory-resistive systems [2]. We also provide a simple analytical example of memristive behavior which has been employed in describing "learning circuits" [10]. For a broad range of memristive system models, see, e.g., Ref. [14].

Definition - From Eqs. (1) and (2), an $n$ th-order currentcontrolled memristive system is described by the equations

$$
\begin{aligned}
V_{M}(t) & =R(x, I, t) I(t) \\
\dot{x} & =f(x, I, t)
\end{aligned}
$$

with $x$ a vector representing $n$ internal state variables, $V_{M}(t)$ and $I(t)$ denote the voltage and current across the device, and $R$ is a scalar, called the memristance (for memory resistance) with the physical units of $\mathrm{Ohm}$. The equation for a chargecontrolled memristor is a particular case of Eqs. (3) and (4), when $R$ depends only on charge, namely

$$
V_{M}=R(q(t)) I
$$

with the charge related to the current via time derivative: $I=$ $d q / d t$. Note that the analytical equation describing the $\mathrm{TiO}_{2}$ device derived in the work by the Hewlett-Packard group [7] has precisely this form, and it therefore represents an ideal memristor.

We can also define an $n$ th-order voltage-controlled memristive system from the relations

$$
\begin{aligned}
I(t) & =G\left(x, V_{M}, t\right) V_{M}(t) \\
\dot{x} & =f\left(x, V_{M}, t\right)
\end{aligned}
$$

where we call $G$ the memductance (for memory conductance).

Properties - Several properties of memristive systems were identified by Chua and Kang in their seminal paper [2]. Here, we highlight just a few of them. Considering devices with $R(x, I, t)>0$ in Eq. (3), it has been proven that these devices are passive [2]. Another important feature, following from the above inequality, is the so-called "no energy discharge property" which is related to the fact that a memristive system can not store energy, like a capacitor or an inductor. As a manifestation of these characteristics, we notice from Eq. (3) [or Eq. [6] that $V_{M}=0$ whenever $I=0$ (and vice versa). In addition, for a periodic current input, the memristive system shows a "pinched hysteretic loop" [2].

Moreover, a memristive system behaves as a linear resistor in the limit of infinite frequency and as a non-linear resistor in the limit of zero frequency provided that $\dot{x}=f(x, I)=$ 0 in Eq. (4) (respectively $\dot{x}=f\left(x, V_{M}\right)=0$ in Eq. (7)) has a steady-state solution. These two properties are easy to understand on physical grounds. Irrespective of the underlying physical mechanisms that define the state of the system, at very low frequencies, the system has enough time to adjust its value of resistance to a momentary value of the control parameter (either current or voltage), so that the device behaves as a non-linear resistor (from Eq. (3) or Eq. (6)). On the other hand, at very high frequency, there is not enough time for any kind of resistance change during a period of oscillations of the control parameter, so that the device operates as a usual (linear) resistor.

Finally, it is worth pointing out that, when a current flows in a resistor, at the microscopic level local resistivity dipoles form due to scattering of carriers at interfaces [15]. Namely, charges accumulate on one side of the resistor with consequent depletion on the other side, with possible accumulation and depletion over the whole spatial extent of the resistor [16]. These dipoles develop dynamically over length scales of the order of the screening length [17], namely within length scales over which electrons can effectively screen the electric field of a local charge. In other words, the self-consistent formation of local resistivity dipoles takes some time and is generally accompanied by some energy storage related to the capacitance of the system. We thus expect that in nanoscale systems memristive behavior is always accompanied to some extent by a (possibly very small) capacitative behavior and as such the hysteresis loop may not exactly cross the origin at frequencies comparable to the inverse characteristic time of charge equilibration processes. This is, for instance, found in the transverse voltage response of the spin Hall effect in inhomogeneous semiconductor systems [18].

Examples - Several systems have been found to satisfy the above properties [1], [2], [3], [7], [8], [5], [9], and many more are likely to be found which fit this classification. In particular, memristive behavior is a property of thermistors [3], molecular systems [4], spintronic devices [5] and thin film nanostructures [7], [8], [9], as well as numerous examples presented in Ref. [14].

As an example, let us consider a model of a voltagecontrolled memristive system used by two of the present authors (MDV and YVP) in Ref. [10]. In this model, the rate of system resistance change ( $R$ changes between two limiting values $R_{1}$ and $R_{2}$ ) is characterized by a constant $\alpha$ when $\left|V_{M}\right| \leq V_{T}$ and $\beta$ when $\left|V_{M}\right|>V_{T}$, where $V_{M}$ is the voltage applied to the memristor and $V_{T}$ is a threshold voltage [19]. Mathematically, the response of this memristive system is described by Eqs. (6, 7) with

$$
\begin{aligned}
G= & x^{-1}, \\
\dot{x}= & -\left(\beta V_{M}+0.5(\alpha-\beta)\left[\left|V_{M}+V_{T}\right|-\left|V_{M}-V_{T}\right|\right]\right) \\
& \times\left[\theta\left(V_{M}\right) \theta\left(x-R_{1}\right)+\theta\left(-V_{M}\right) \theta\left(R_{2}-x\right)\right]
\end{aligned}
$$




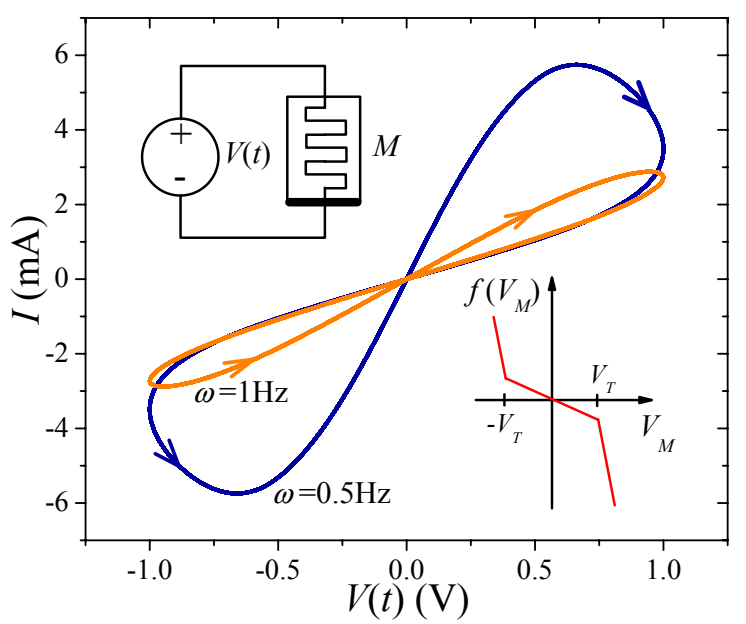

Fig. 2. (Color online) Simulation of a voltage-controlled memristive system. This plot was obtained by a numerical solution of Eqs. 699 for the case of a memristive system $M$ connected directly to an alternating (ac) voltage source $V(t)=V_{0} \sin (2 \pi \omega t)$ as shown in the inset using the following parameter values: $R_{1}=20 \Omega, R_{2}=500 \Omega, V_{T}=0.5 \mathrm{~V}, \alpha=-500 \mathrm{~V} /(\Omega \cdot \mathrm{s}), \beta=2 \alpha$, $V_{0}=1 \mathrm{~V}$. In our model, the rate of resistance change is defined by the function $f\left(V_{M}\right)=-\left(\beta V_{M}+0.5(\alpha-\beta)\left[\left|V_{M}+V_{T}\right|-\left|V_{M}-V_{T}\right|\right]\right)$ entering Eq. (9) and schematically shown in the inset for positive values of $\alpha$ and $\beta$. At $\left|V_{M}\right|<V_{T}$ the slope of $f\left(V_{M}\right)$ is determined by $\alpha$ and at $\left|V_{M}\right|>V_{T}$ it is determined by $\beta$.

where $\theta(\cdot)$ is the step function. The simulation results [20] depicted in Fig. 2 clearly show a pinched hysteresis loop and a hysteresis collapse with increasing frequency $\omega$ of the alternating (ac) voltage source $V(t)=V_{0} \sin (2 \pi \omega t)$.

We can now extend the above definitions to capacitances and inductances. The memory devices that result share many characteristics of memristive systems, but with a fundamental difference: they store energy.

\section{Memcapacitative Systems}

Definition - We define an $n$ th-order voltage-controlled memcapacitive system by the equations

$$
\begin{aligned}
q(t) & =C\left(x, V_{C}, t\right) V_{C}(t) \\
\dot{x} & =f\left(x, V_{C}, t\right)
\end{aligned}
$$

where $q(t)$ is the charge on the capacitor at time $t, V_{C}(t)$ is the corresponding voltage, and $C$ is the memcapacitance (for memory capacitance) which depends on the state of the system. Similarly, we can define an $n$ th-order chargecontrolled memcapacitive system from the equations

$$
\begin{aligned}
V_{C}(t) & =C^{-1}(x, q, t) q(t) \\
\dot{x} & =f(x, q, t)
\end{aligned}
$$

where $C^{-1}$ is an inverse memcapacitance.

In addition, we define a subclass of the above devices, we call voltage-controlled memcapacitors, when Eqs. (10) and (11) reduce to

$$
q(t)=C\left[\int_{t_{0}}^{t} V_{C}(\tau) d \tau\right] V_{C}(t)
$$

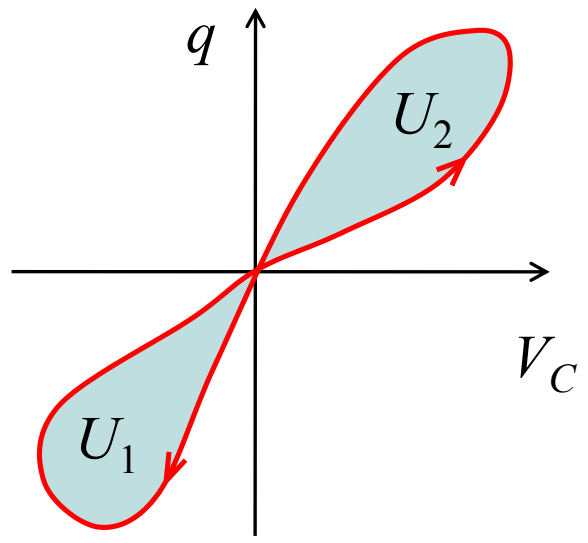

Fig. 3. (Color online) Schematics of a pinched hysteresis loop of a memcapacitive system. The energy added to/removed from the system $\left(\int V_{C}(q) d q\right)$ is the area between the curve and the $q$ axis. The areas of shaded regions $U_{1}$ and $U_{2}$ give the amount of added/removed energy in each half-period. The signs of $U_{1}$ and $U_{2}$ are determined by the direction on the loop. For the direction shown here, $U_{2}$ is positive and $U_{1}$ is negative.

and a charge-controlled memcapacitor when Eqs. (12) and (13) can be written as

$$
V_{C}(t)=C^{-1}\left[\int_{t_{0}}^{t} q(\tau) d \tau\right] q(t),
$$

where in the above two equations, the lower integration limit (initial moment of time) may be selected as $-\infty$, or 0 if $\int_{-\infty}^{0} V_{C}(\tau) d \tau=0$ and $\int_{-\infty}^{0} q(\tau) d \tau=0$, respectively.

Properties - It follows from Eq. (10) that the charge is zero whenever the voltage is zero. Note, however, that in this case, $I=0$ does not imply $q=0$, and thus this device can store energy. Therefore, the latter can be both added to and removed from a memcapacitive system.

From a microscopic point of view a change in capacitance can occur in two ways: $i$ ) due to a geometrical change of the system (e.g., a variation in its structural shape), or ii) in the quantum-mechanical properties of the carriers and bound charges of the materials composing the capacitor (manifested, e.g., in a history-dependent permittivity $\varepsilon$ ), or both.

In either case, inelastic (dissipative) effects may be involved in changing the capacitance of the system upon application of the external control parameter. These dissipative processes release energy in the form of heating of the materials composing the capacitor. However, this heat may not be simply accounted for as a (time-dependent) resistance in series with the capacitor.

Similarly, there may be situations in which energy not from the control parameter but from sources that control the equation of motion for the state variable, Eq. (11), is needed to vary the capacitance of the system (e.g., in the form of elastic energy or via a power source that controls, say, the permittivity of the system via a polarization field). This energy can then be released in the circuit thus amplifying the current. Therefore, Eqs. (10) and (11) for memcapacitive systems postulated above could, in principle, describe both active and passive devices. 
However, starting from a fully discharged state, the amount of removed energy from a passive memcapacitive system can not exceed the amount of previously added energy. Mathematically, this property can be written in the form

$$
U_{C}=\int_{t_{0}}^{t} V_{C}(\tau) I(\tau) d \tau \geq 0
$$

under the condition that at $t=t_{0}$ no energy is stored in the system. Eq. (16) should be valid for any form of the control parameter, such as the voltage $V_{C}(t)$ applied to the passive memcapacitive system. Moreover, in memcapacitive systems with dissipative processes (such as heating), the equality sign in Eq. (16) for $t>t_{0}$ is not realized (assuming $V_{C}(t) \neq 0$ ).

Fig. 3 shows a schematic memcapacitive system hysteresis loop passing through the origin. The shaded areas give the energy $U_{i}$ added to/removed from the system. Note that this energy is associated with some internal degree of freedom, i.e., related to some physical elastic or inelastic processes that accompany the change in conductance. The system is passive if $U_{1}+U_{2}=0$, dissipative if $U_{1}+U_{2}>0$ and active if $U_{1}+U_{2}<0$.

Finally, since the state equation (11) has (by assumption) only a unique solution at any given time $t \geq t_{0}$, then if $V_{C}(t)$ is periodic with frequency $\omega$, namely $V_{C}(t)=V_{0} \cos (2 \pi \omega t)$, then the $q-V_{C}$ curve is a simple loop passing through the origin, namely there may be at most two values of the charge $q$ for a given voltage $V_{C}$, if we consider a voltage-controlled device (see Fig. (4), or two values of the voltage $V_{C}$ for a given charge $q$, for a charge-controlled system. This loop is also anti-symmetric with respect to the origin if, for the case of Eq. (10]11), $C\left(x, V_{C}, t\right)=C\left(x,-V_{C}, t\right)$ and $f\left(x, V_{C}, t\right)=$ $f\left(x,-V_{C}, t\right)$.

Like the case of memristive systems, a memcapacitive system behaves as a linear capacitor in the limit of infinite frequency, and as a non-linear capacitor in the limit of zero frequency, assuming Eqs. (11) and (13) admit a steady-state solution. The origin of this behavior rests again on the system's ability to adjust to a slow change in bias (for low frequencies) and the reverse: its inability to respond to extremely high frequency oscillations.

Examples - There are instances in which the capacitance $C$ has been found to have a hysteresis-type dependence on the applied voltage [21], [22], [23]. These cases are related to nanoscale capacitors in which interface traps [21] or embedded nanocrystals [22], [23] are responsible for memory effects. Even though characteristic hysteresis properties of these systems were not discussed in the framework of the memcapacitive systems suggested in this paper, it is clear that they belong to the present classification.

Below, we provide a model example of a memcapacitive system which could be used to simulate experimental realizations of memcapacitors. To be specific, let us consider a voltage-controlled memcapacitive system according to Eqs. (10) and (11). We focus on the electronic circuit shown in the inset of Fig. 4. This scheme consists of a memcapacitive system $C$ connected to a source of ac-voltage $V(t)=V_{0} \sin (2 \pi \omega t)$. The capacitance $C$ is chosen to vary in
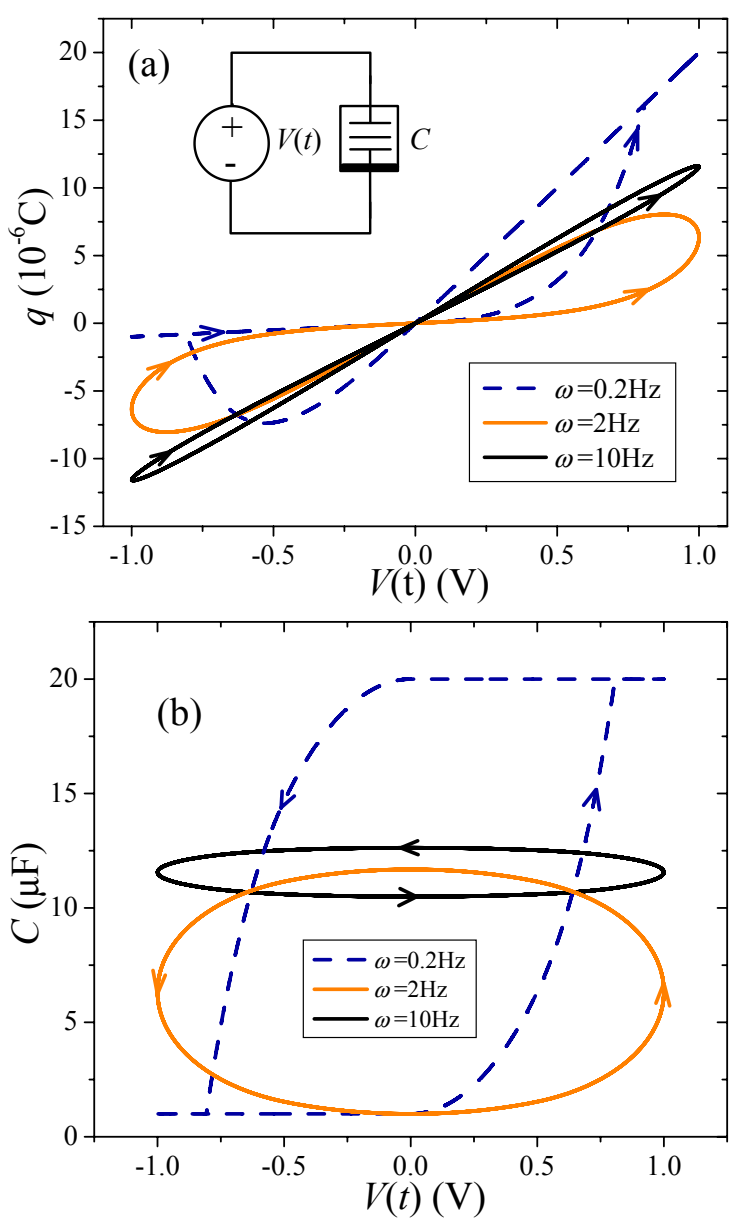

Fig. 4. (Color online) Simulation of a circuit (shown in the inset of (a)) composed of a voltage-controlled memcapacitive system and a resistance. The following parameter values were used in this calculation: $C_{1}=1 \mu \mathrm{F}$, $C_{2}=20 \mu \mathrm{F}, V_{T}=0.5 \mathrm{~V}, \alpha=-50 \mu \mathrm{F} /(\mathrm{V} \cdot \mathrm{s}), \beta=2 \alpha, V_{0}=1 \mathrm{~V}$.

the range between its minimum and maximum values $\left(C_{1}\right.$ to $C_{2}$ ) and changes according to the equations

$$
\begin{aligned}
C= & x \\
\dot{x}= & -\left(\beta V_{C}+0.5(\alpha-\beta)\left[\left|V_{C}+V_{T}\right|-\left|V_{C}-V_{T}\right|\right]\right) \\
& \times\left[\theta\left(V_{C}\right) \theta\left(x-C_{1}\right)+\theta\left(-V_{C}\right) \theta\left(C_{2}-x\right)\right] .
\end{aligned}
$$

Here, $V_{C}$ is the voltage drop on the memcapacitive system, $V_{T}$ is a threshold voltage, $\alpha$ is a variation rate for $\left|V_{C}\right|<V_{T}$ and $\beta$ is a variation rate for $\left|V_{C}\right|>V_{T}$. The interpretation of the coefficients $\alpha$ and $\beta$ is the same as in Fig. 2] The response of the circuit is simply described by the equation $V(t)=V_{C}=q / C$ where $C$ can be found using Eqs. (17/18). It is important to remember that in modeling of more complex circuits the chain rule of differentiation [24] should be used.

Fig. 4 represents results of our numerical simulations. Typical features discussed above such as capacitance hysteresis, low-frequency and high-frequency behavior, and pinched hysteresis loop can be clearly distinguished. We also checked the energy conservation for the given circuit and found that for the present model the memcapacitive system behaves as a passive device. 


\section{Meminductive Systems}

Let us now introduce the third class of memory devices. Let us define the "flux"

$$
\phi(t)=\int_{-\infty}^{t} V_{L}\left(t^{\prime}\right) d t^{\prime}
$$

where $V_{L}(t)$ is the induced voltage on the inductor (equal to minus the electromotive force).

Definition - We call an $n$ th-order current-controlled meminductive system one described by the equations

$$
\begin{aligned}
\phi(t) & =L(x, I, t) I(t) \\
\dot{x} & =f(x, I, t)
\end{aligned}
$$

where $L$ is the meminductance, and an $n$ th-order fluxcontrolled meminductive system the following

$$
\begin{aligned}
I(t) & =L^{-1}(x, \phi, t) \phi(t) \\
\dot{x} & =f(x, \phi, t)
\end{aligned}
$$

with $L^{-1}$ the inverse meminductance.

We call current-controlled meminductors a subclass of the above devices, when Eqs. (20) and (21) reduce to

$$
\phi(t)=L\left[\int_{t_{0}}^{t} I(\tau) d \tau\right] I(t)
$$

and a flux-controlled meminductor when Eqs. 221 and 23 can be written as

$$
I(t)=L^{-1}\left[\int_{t_{0}}^{t} \phi(\tau) d \tau\right] \phi(t) .
$$

In the above two equations, the lower integration limit may be selected as $-\infty$, or 0 if $\int_{-\infty}^{0} I(\tau) d \tau=0$ and $\int_{-\infty}^{0} \phi(\tau) d \tau=$ 0 , respectively.

Properties - For the sake of definiteness, we shall consider the current-controlled meminductive systems. Taking the time derivative of both sides of Eq. (20) yields

$$
V_{L}=\frac{\mathrm{d} \phi}{\mathrm{d} t}=L \frac{\mathrm{d} I}{\mathrm{~d} t}+I \frac{\mathrm{d} L}{\mathrm{~d} t} .
$$

The second term on the right-hand side of the above equation is an additional contribution to the induced voltage due to a time-dependent $L$. The energy stored in the current-controlled meminductive system can be thus calculated as

$$
U_{L}(t)=\int_{t_{0}}^{t} V_{L}(\tau) I(\tau) d \tau=\int_{t_{0}}^{t}\left[L \frac{\mathrm{d} I}{\mathrm{~d} t}+I \frac{\mathrm{d} L}{\mathrm{~d} t}\right] I(\tau) d \tau .
$$

When $L$ is constant, we readily obtain the well-known expression for the energy $U_{L}=L I^{2} / 2$. Usually, this $U_{L}$ is interpreted as the energy of the magnetic field generated by the current. Similarly to the passivity criterion of memcapacitive systems (see Eq. (16) Eq. (27) provides the passivity criterium of meminductive system if at $t=t_{0}$ the latter is in its minimal energy state and $U_{L}(t) \geq 0$ at any time.

Finally, as in the case of memristive and memcapacitive systems, it is expected that when the external control parameter is a periodic function of time with frequency $\omega$, namely $I(t)=$ $I_{0} \cos (2 \pi \omega t)$, the $\phi-I$ curve is a simple loop passing through the origin, namely there may be at most two values of the flux $\phi$ for a given current $I$. This loop is anti-symmetric with respect to the origin if, for the case of Eq. (20)21), $L(x, I, t)=$ $L(x,-I, t)$ and $f(x, I, t)=f(x,-I, t)$.

Also, the magnetic permeability can adjust to slow periodic variations of the current, while it cannot at high frequencies, so that this device behaves as a non-linear inductor at low frequencies (assuming Eqs. (21) and (23) admit a steady-state solution) and a linear inductor at high frequencies.

Examples - In electronics, inductors are primarily of a solenoid type consisting of a coil of conducting material wrapped around a core. The inductance of a solenoid is proportional to the relative permeability $\mu_{r}$ of the material within the solenoid and also depends on the geometrical parameters of the system. The simplest way to introduce a memory effects in such a system is to use the core material whose response to the applied magnetic field depends on its history. As an example, we can think about ferromagnetic materials exhibiting a magnetic hysteresis such as iron. In fact, an electronic circuit with such inductor was recently analyzed [25]. Another way to introduce memory effects is by varying the inductor's shape.

A circuit model for a meminductive system can be formulated similarly to the models of memristive and memcapacitive systems discussed above. In particular, we can imagine a structure whose inductance increases up to a certain value when current flows in one direction and decreases down to another value if the current flow direction is reversed. This is a straightforward calculation which does not add much to the analysis of this problem. Therefore, we do not report it here.

\section{CONCLUSions}

We have extended the notion of memory devices to both capacitative and inductive systems. These devices have specific properties that appear most strikingly as a pinched hysteretic loop in the two constitutive variables that define them: currentvoltage for memristive systems, charge-voltage for memcapacitive systems, and current-flux for the meminductive systems. Many systems belong to these classifications, especially those of nanoscale dimensions. Indeed, with advances in the miniaturization of devices, these concepts are likely to become more relevant since at the nanoscale the dynamical properties of electrons and ions may strongly depend on the history of the system, at least within certain time scales [16].

Apart from the obvious use of these devices in non-volatile memories, several applications can be already envisioned for these systems, especially in neuromorphic devices to simulate learning, adaptive and spontaneous behavior. For instance, the identification of memristive behavior in primitive organisms such as amoebas [10], opens up the possibility to relate physiological processes that occur in cells with the theory of 
memory devices presented here. Along similar lines, one could envision simple models that identify memory mechanisms in neurons and use these memory devices to build such models in the laboratory.

Therefore, due to their versatility (including analog functionalities) the combined operations of these memory devices in electronic circuits is still largely unexplored, and we hope our work will motivate experimental and theoretical investigations in this direction.

\section{ACKNOWLEDGMENT}

The authors are indebted to B. Mouttet for pointing out Ref. [22]. This work has been partially funded by the NSF grant No. DMR-0802830.

\section{REFERENCES}

[1] L. O. Chua, "Memristor - The Missing Circuit Element," IEEE Trans. Circuit Theory, vol. 18, pp. 507-519, 1971

[2] L. O. Chua and S. M. Kang, "Memrisive devices and systems," Proc. IEEE, vol. 64, pp. 209-223, 1976.

[3] M. Sapoff and R. M. Oppenheim, "Theory and application of selfheated thermistors," Proc. IEEE, vol. 51, pp. 1292-1305, 1963.

[4] Y. Chen, G. Y. Jung, D. A. A. Ohlberg, X. M. Li, D. R. Stewart, J. O. Jeppesen, K. A. Nielsen, J. F. Stoddart, and R. S. Williams, "Nanoscale molecular-switch crossbar circuits," Nanotech., vol. 14, pp. 462-468, 2003.

[5] Yu. V. Pershin and M. Di Ventra, "Spin memristive systems: Spin memory effects in semiconductor spintronics," Phys. Rev. B, Condens. Matter, vol. 78, p. 113309/1-4, 2008.

[6] Yu. V. Pershin and M. Di Ventra, "Current-voltage characteristics of semiconductor/ferromagnet junctions in the spin-blockade regime," Phys. Rev. B, Condens. Matter vol. 77, p.073301/1-4, 2008; "Spin blockade at semiconductor/ferromagnet junctions," Phys. Rev. B, Condens. Matter, vol. 75, p. 193301/1-4, 2007.

[7] D. B. Strukov, G. S. Snider, D. R. Stewart, and R. S. Williams, "The missing memristor found," Nature (London), vol. 453, pp. 80-83, 2008.

[8] J. J. Yang, M. D. Pickett, X. Li, D. A. A. Ohlberg, D. R. Stewart, and R. S. Williams, "Memristive switching mechanism for metal/oxide/metal nanodevices," Nature Nanotechnology, vol. 3, pp. 429-433, 2008.

[9] T Driscoll, H.-T. Kim, B.-G. Chae, M. Di Ventra, and D. N. Basov, "Phase-transition driven memristive system," arXiv:0901.0899. 2009.

[10] Yu. V. Pershin, S. La Fontaine and M. Di Ventra, "Memristive model of amoeba's learning," arXiv:0810.4179 2008.

[11] L. O. Chua, "Nonlinear Circuit Foundations for Nanodevices, Part I: The Four-Element Torus," Proc. IEEE, vol. 91, pp. 1830-1859 (2003).

[12] Hypothetical choice of some non-generic non-linear function $f(x, u, t)$ in Eq. 22) could give rise to non-unique solutions (see, e.g., p. 1063 in Ref. [13]).

[13] L. O. Chua, Dynamic nonlinear networks: state-of-the-art, IEEE Trans. Circuits and Systems, vol. 11, pp. 1059-1087, 1980.

[14] L. O. Chua, "Introduction to memristors," IEEE Expert Now Educational Course.

[15] R. Landauer, "Spatial Variation of Currents and Fields Due to Localized Scatterers in Metallic Conduction," IBM J. of Research and Development, vol. 1, pp. 223-231, 1957.

[16] M. Di Ventra, Electrical Transport in Nanoscale Systems (Cambridge University Press, Cambridge, 2008)

[17] N. Sai, N. Bushong, R. Hatcher and M. Di Ventra, "Microscopic current dynamics in nanoscale junctions," Phys. Rev. B, Condens. Matter, vol. 75, p. 115410/1-8, 2007.

[18] Yu. V. Pershin and M. Di Ventra, "Frequency doubling and memory effects in the Spin Hall Effect," arXiv:0812.4325, 2008.

[19] This model was inspired by an activation-type resistance state found in nanostructure memristors [8].

[20] All numerical calculations presented in this paper have been performed using a fourth-order Rugge-Kutta integration method.

[21] D. M. Fleetwood, M. R. Shaneyfelt, W. L. Warren, J. R. Schwank, T. L. Meisenheimer, and P. S. Winokur, "Border Traps: Issues for MOS Radiation Response and Long-Term Reliability," Microel. Reliab., vol. 35, pp. 403-428, 1995.
[22] Y. Kim, K. H. Park, T. H. Chung, H. J. Bark, J.-Y. Yi, W. C. Choi, E. K. Kim, J. W. Lee, and J. Y. Lee, "Ultralarge capacitancevoltage hysteresis and charge retention characteristics in metal oxide semiconductor structure containing nanocrystals deposited by ion-beam-assisted electron beam deposition," Appl. Phys. Lett., vol. 78, pp. 934-936, 2001.

[23] P. F. Lee, X. B. Lu, J. Y. Dai, H. L. W. Chan, E. Jelenkovic, and K. Y. Tong, "Memory effect and retention property of Ge nanocrystal embedded Hf-aluminate high-k gate dielectric," Nanotechn., vol. 17, pp. 1202-1206, 2006.

[24] In the case of a memory capacitor, since the capacitance $C$ is timedependent the well-known relation $\mathrm{d} q=C \mathrm{~d} V_{C}$ generalizes to $\mathrm{d} q=$ $C \mathrm{~d} V_{C}+V_{C} \mathrm{~d} C$

[25] T. Matsuo, K. Okumura, and A. Kishima, "Analysis of a hysteretic circuit containing an iron-cored inductorand a semiconductor switch," IEE Proc. Circ. Dev. and Syst., vol. 146, pp. 176-183, 1999. 